\title{
Characterization of HVAC Operation Uncertainty in EnergyPlus AHU Modules
}

\author{
Di Sui ${ }^{1}$, Yuna Zhang ${ }^{2}$, Godfried Augenbroe ${ }^{3}$ \\ ${ }^{1}$ Center for Energy and Environment, Minneapolis, MN, U.S.A. \\ ${ }^{2}$ Baumann Consulting, Washington, DC, U.S.A \\ ${ }^{3}$ College of Architecture, Georgia Institute of Technology, Atlanta, GA, U.S.A
}

\begin{abstract}
The energy impact of building performance simulation uncertainties are often carried out using existing simulation tools, with Monte Carlo method applied to sample the possible parameter uncertainty combinations. The results of those analyses are useful in justifying the importance of uncertainty analysis and quantifying the impact of simulation uncertainties in a general way. However, those results are not practical enough to provide assistance in actual building designs. This paper introduces a way to implement the uncertainty analysis by modifying the source code of a commonly used simulation tool - EnergyPlus so that the updated tool can be used for everyday building energy simulation works.
\end{abstract}

\section{Introduction}

Building energy simulation tools have been widely used to facilitate design for new constructions and support evaluation and renovation for existing buildings. However, significant deviations in terms of building energy consumption can be observed between modelpredicted results and building meter data. Deviations in energy consumption between predicted and actual building can be attributed to simulation uncertainties introduced by three components in our predictions: the accuracy of the underlying models in simulation tools, the actual weather, and the accuracy of input parameters describing the design conditions (Wang et.al, 2012). To get a better energy simulation result, those deviations should be captured and taken into consideration. In this study we focus on how to improve the accuracy of simulation models. As one major part of a building, HVAC system is considered as an important simulation object in building energy modelling since it is the bridge of converting "the amount of energy required to keep the building performing in a comfortable way" to "the amount of source energy that systems consume for running the building". Advanced simulation tools such as EnergyPlus represent HVAC systems as truthfully as possible, taking all important parameters into consideration. Inevitably, energy models assume that every part of the system works as directed by the schedule and under ideal conditions. This is obviously not the case, and deviations between ideal and actual operation will contribute to the performance gap, i.e. the difference between simulated and measured energy consumption. Simulation results can become more reliable if all deviations between ideal and actual behaviour can be predicted and taken into consideration.
Studies have been carried out to capture those deviations using different viewpoints. One viewpoint is to directly capture in a non-intrusive way the differences observed in real buildings. Typically one can build a Gaussian Process model with measured training data, and then use the model with new inputs to make predictions about uncertainty in outcomes (Yan \& Malkawi, 2012). Despite that this method doesn't require the model itself to be accurate, it can only relatively well predict the building energy consumption on condition that groups of reliable training data are accessible. In our study we are interested in the prediction at the design stage. In that case we can only rely on intrusive methods of capturing the uncertainties at the model level. Therefore, detailed causes of deviations should be embedded in module modifications in the HVAC system modules of the simulation software. In this study, uncertainties of 5 specific parameters in AHU operation are introduced. The simulation used for this analysis is EnergyPlus.

HVAC systems can deviate from their intended behaviour by two major causes. The first one is called "fault" (M. Basarkar et.al, 2011), which shows as equipment fails or faulty operation (e.g. temperature sensor that suddenly fails or drifting over time). The other cause is that even when the building equipment is new and operating normally, most of the time it will not perform ideally as expected. For instance, a damper may open to $91 \%$ or $89 \%$ when it is told to open $90 \%$. We call this type of cause "operation uncertainty". This paper only focuses on the second cause, i.e. uncertainties in HVAC system operation. To make it possible to add operation uncertainties as a part of the model in a simulation, detailed possible causes of uncertainties are identified and added into EnergyPlus source code to change the values of corresponding system parameters.

\section{Building Model Description}

The DOE commercial building reference model in compliance with ASHRAE 90.1-2010 for a large office building located in Atlanta is used as the baseline for the study. The building is mainly served by only one type of HVAC system: air-based heating and cooling by air handling unit (AHU) with VAV terminal boxes, using boiler and chiller as plants. The reason why we choose this type of system is that it is the most widely used one for commercial buildings. The building has four stories with one basement and three upper floors. The basement has one thermal zone and each upper level has 5 zones (four perimeter zones and one core zone). Each floor is served by one variable volume AHU with VAV reheat terminal boxes in the zones. In each AHU there's one 
heating coil and one cooling coil. Each VAV terminal box has one reheat coil. In total there are 20 heating coils in the system. Each AHU also has an OA damper and is controlled by an economizer. The building has one boiler that serves hydronic hot water to the building, and two chillers which together provide chilled water.

\section{Selected Uncertainty Parameters}

There is no prior work on encapsulating operation uncertainty in HVAC components at the level of the empirical models in EnergyPlus. Nor is there a theory that would let us identify the minimal and useful set of parameters that would sufficiently capture the deviations from ideal behaviour. This section postulates a number of parameters based on the fact that certain physical states of the AHU are hard to control in reality. Each of these physical states is then reduced to a postulated parameter that can be positioned in the EnergyPlus modules that make up the AHU. The HVAC system of the baseline building consists of three major parts: 1) primary air loop which includes heating and cooling coils in AHU, outdoor air controller and mixture, and supply air fan. 2) Zone equipment loop which includes the zone VAV boxes and associated components. 3) the plant loop which includes chiller, boiler and other associated components. Five uncertainties are identified: heating coil UA factor uncertainty located in heating coils, OA controller uncertainty located in outdoor air controller, temperature sensor uncertainty located at the outlet of heating coil in AHU, water distribution uncertainty located in the distribution of heating and cooling water that supplied into coils, and air distribution uncertainty located in the distribution of supply air. Each of these will be treated in detail below.

\section{System UQ: OA Controller}

In a common building HVAC system, the mass flow rate of outdoor air (OA) is calculated based on system requirements and environment conditions. This result will then be converted into a damper opening signal and passed to the OA damper. As we all know, most dampers are not accurate enough to bring in the exact amount of air as we expect in the ideal case. However, this damper uncertainty has not been taken into consideration in the commonly used simulation tools.

Two steps are taken in order to implement the damper uncertainty into EnergyPlus: (1) Figuring out where to insert the uncertainty, and (2) Figuring out how to model the uncertainty.

For step 1, the EnergyPlus airflow network simulation process is illustrated in Figure 1 based on the analysis of EnergyPlus source code. The target parameter that needs to be modified is the OA flow rate calculated by module "OA controller", which is named as "OAMassFlow" in the source code. The target parameter should be modified directly after it is calculated by "OA controller" so that the new outdoor air mass flow rate will impact the relief air mass flow rate and keep the total mixed air mass flow rate unchanged in "OA Mixer".

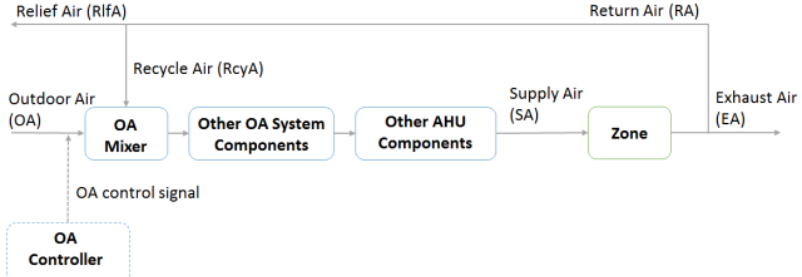

Figure 1: Air flow network.

After figuring out the target parameter, in step 2 a new parameter named "OADamperUQValue" is defined to represent the OA damper uncertainty value. The new "OAMassFlow" is then calculated with Equation (1) below.

$$
\begin{aligned}
& \text { OAController\%OAMassflow } \\
& =(1+\text { OADamperUQValue }) \\
& \times \text { OAController\%OAMassflow }
\end{aligned}
$$

This equation was not added into the original module but added as a new UQ module. During each execution of module "OA Controller", this UQ module will be called to update the OA mass flow rate at the spot described above in step 1. Before the update, the UQ module will have to first generate a uncertainty value named "OADamperUQValue". This value will vary in every time step, and from damper to damper. The uncertainty values for each damper are generated based on uniform distribution with a pair of symmetric upper and lower bounds which are defined as user inputs (+/- 10\% are used for this paper). If the generated value is positive, the module "OA controller" will pass a larger OA mass flow rate value then the ideal one (original result) to the OA Mixer; If negative, the output of module "OA Controller" will be smaller then it's original result.

\section{System UQ: Heating Coil UA}

In EnergyPlus, object "Coil: Heating: Water" provides a module that uses an NTU-effectiveness model of a static heat exchanger. The model takes UA, the effectiveness, as well as inlet fluids conditions and flow rates as its inputs and calculates the outlet fluids conditions as outputs (Zhang, 2001). Inlet fluids include inlet hot water and inlet air that ready to be heated up. The UA is either a user input or calculated based on the design conditions of the heating coil. No matter how the UA is assigned, the value of this parameter is fixed and will not be changed during the whole simulation. However, in a real system, the UA value for most coils are not constantly the same as their labeled values due to imprecisely manufacture, and the real UA of a coil in use may not equal to the rated value if the coil is not fully loaded.

The implement of UA could be performed in two possible ways:

1. Consider the uncertainty as an inherent attribute of each coil and add a user defined parameter for the coil module as a ratio of how good the target heating coil can performance as a heat exchanger.

2. Consider the uncertainty as a function of the hot water flow rate and modify the UA value in the simulation in every time step using this function. 
For this paper, since it's hard to figure out the relation between UA value and hot water flow rate, the first method is used to estimate the uncertainty.

The logic of heating coil simulation is quite simple and straight forward in EnergyPlus. The Subroutine "SimulateWateCoilComponents" will be called for simulating the heating coil, and the target parameter that needs to be modified in this subroutine is called "UACoilVariable". A parameter named "HeatingCoilUAUncertaintyFactor" is added into the heating coil module to represent the uncertainty value and will be defined as a user input. Once the uncertainty value is assigned, it will be fixed for the rest of the simulation.

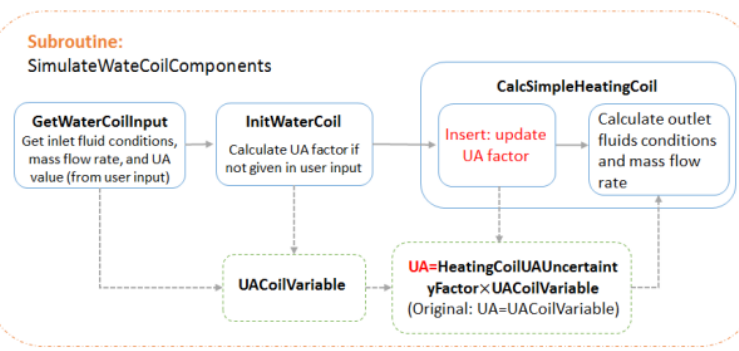

Figure 2: Working Process of Subroutine "SimulateWaterCoilComponents".

Figure 2 above shows the process of the modified subroutine. The target parameter "UACoil Variable" is modified with Equation (2).

\section{WaterCoil\%UACoilVariable $=$ HeatingCoilUAUncertaintyFactor $\times$ WaterCoil\%UACoilVariable}

\section{System UQ: Temperature Controller}

Figure 3 illustrates the principle of a mostly used simple water coil controller. The controller reads the real outlet air temperature (sensed value) as well as the desire outlet air setpoint temperature (setpoint value) from the control node and maintain the sensed temperature at the setpoint value by modulating the inlet water flow rate at the actuator node.

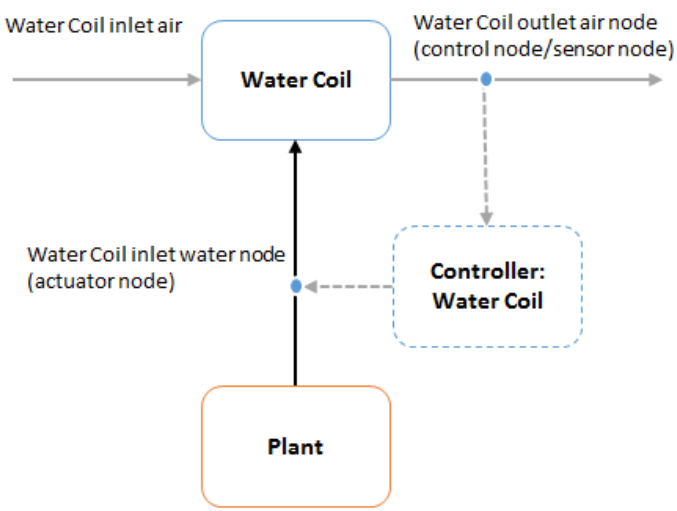

Figure 3: Work process of controller: Water Coil.
According to the principle above, EnergyPlus does the calculation below to find out the proper water flow rate:

1. Read the outlet air temperature "SensedValue",

2. Calculate the difference between the sensed value and setpoint value "DeltaSensed"

3. Use root-finding algorithm to find the "NextActuatedValue" such that the corresponding "DeltaSensed" value equals to 0 .

While in reality, the sensed temperature will not be the same as its actual value due to the sensor uncertainty, which will cause the coil controller to come up with a different water inlet flow rate, and will impact both the plant energy consumption and the coil outlet air temperature.

For now, we consider this uncertainty as an inherent attribute of the sensor, which means the uncertainty factor will be set up only once and used for the entire simulation. (e.g. if the factor $>0$, the sensor will always give a larger number then the actual value.) To capture this accuracy of the sensor, we add a module named "Temperature Controller". It allows user to define a value to represent the sensor inaccuracy. By changing the code of controller simulation, this user input factor will be used to add uncertainty to the target parameter "Sensed Value".

SensedValue

$=$ Node(sensedNode)\%Temperature

$\times$ TemperatureControllerUncertaintyFactor

\section{System UQ: Hot Water Distribution in Air Handling Unit and System UQ: Water Distribution in VAV Reheat Coils}

For each air loop served by one AHU, there are more than one heating coil located in the air handling unit or in each VAV terminal boxes (re-heat coil). All those coils get their inlet hot water from one plant with the same pump. Thus, both the inaccuracy of the pump and the unbalance of water network will cause the uncertainty of inlet water flow rate for each heating coil.

The two types of uncertainty are both captured with the following equation:

$$
\dot{m}_{i}^{r}=\left(\dot{m}_{i}^{d}+x_{i} \dot{M}^{d}\right)(1+\rho)
$$

where

$\dot{m}_{i}^{r}$ : Actual water mass flow rate received by terminal I

$\dot{m}_{i}^{d}$ : Demand water mass flow rate by terminal I, which is also the target parameter for this uncertainty

$\dot{M}^{d}$ : Total demand water mass flow rate by one pump

$\rho$ : Uncertainty factor that capture the error in the use of idealized pump reaction

$x_{i}$ : Uncertainty factor caused by unbalance of distribution

In order to implement the uncertainties, we have to (1) Update $\dot{m}_{i}^{d}$ to $\dot{m}_{i}^{r}$ with Equation (4), (2) Calculate and assign $\rho, x_{i}$ to each water coil, and (3) Calculate total actuated water mass flow rate $\dot{M}^{d}$. 
In EnergyPlus source code, simulations of air handling unit coils and VAV reheat terminal coils are carried out in different places, so the uncertainty implementation for those two types of water distribution will be discussed separately.

\section{Hot Water Distribution in Air Handling Unit}

In EnergyPlus source code, the water inlet mass flow rate of each coil is calculated and assigned to the corresponding water coil inlet node by a subroutine called "UpdateController" under section "ManageAirLoops". The value of target parameter $\dot{m}_{i}^{d}$ in this subroutine is called "NextActuatedValue". Based on Equation (4), the target parameter is modified with Equation (5).

\section{NextActuatedValue}

$$
\begin{aligned}
& =(\text { NextActuatedValue } \\
& + \text { WaterFlowRateUQ1 } \\
& \times \text { HeatingTotalNextValue }) \\
& \times(1+\text { WaterFlowRateUQ2 })
\end{aligned}
$$

Since subroutine "UpdateControl" is not only used for AHU water coil control, in order to make sure that the modification of the subroutine only affect AHU water coils, a new module named "ReupdateController" which contains the equation above is added to modify the "NextActuatedValue".

WaterFlowRateUQ1 is generated using normal distribution. WaterFlowRateUQ2 is added as a user input and will be read from IDF files. Since once the water system is designed and the pump is selected, the relationships between each branch are defined and will not be changed if the loop structure stays the same, uncertainty value WaterFlowRateUQ1 and WaterFlowRateUQ2 should stay the same during the whole simulation. The two uncertainty values are calculated at the very beginning of the water flow distribution simulation process and fixed during the whole simulation. The value of "HeatingTotalNextValue" is calculated by summing up all the water coil inlets.

\section{Water Distribution in VAV Reheat Coils}

The simulation of VAV reheat terminal coils are carried out in section "ManageZoneEquipment". The target parameter of this uncertainty is called "CalculatedSetPoint" which is called by subroutine "ControlCompOutput". Instead of modifying the original subroutine "ControlCompOutput", a new subroutine is added to replace the original one specifically for VAV reheat coil simulation to make sure that the original subroutine still works in other sections of the simulation. The new subroutine is called "ReControlCompOutput". Based on Equation (4), the target parameter for this uncertainty is modified with Equation (6).

$$
\begin{aligned}
& \text { ZoneController\%CalculatedSetPoint } \\
& =(\text { ZoneController\%CalculatedSetPoint } \\
& + \text { TotalReheatWater } \times \text { UQXiReheat }) \\
& \times(1.0 \mathrm{~d} 0+\text { UQRoReheat }) \\
& \times(1.0 \mathrm{~d} 0+\text { AirFlowUQRo })
\end{aligned}
$$

The two uncertainty factors UQXiReheat and UQRoReheat are generated at the beginning of the simulation and are following the same methodologies as those for water distribution in AHU water coils.

The total water flow rate "TotalReheatWater" is calculated by summing up the reheat water flow rate of all terminals.

\section{System UQ: Air Distribution}

The causes and the quantification for uncertainties in air distribution are mainly the same with those for water distribution. Instead of targeting water inlet mass flow rate, in air distribution we target the demand air mass flow rate, which is represented by name "MassFlow" and calculated in subroutine "SimVAV", and the modification of the parameter will follow equation (7).

$$
\begin{aligned}
& \text { MassFlow }=(\text { MassFlow }+ \text { AirFlowUQXi } \\
&\times \text { TotalAirAHU }) \\
& \times(1.0 \mathrm{~d} 0+\text { AirFlowUQRo })
\end{aligned}
$$

The two uncertainty factors AirFlowUQXi and AirFlowUQRo will be generated at the beginning of the simulation and following the same methodologies as those for water distribution.

The total air flow rate "TotalAirAHU" is calculated by summing up the air mass flow rate of all terminals.

\section{Simulation results analysis}

To test how much the five developed uncertainties are going to impact the energy consumption, we used GURA-W (Georgia Tech Uncertainty and Risk Analysis workbench), which was specifically developed to carry out uncertainty analysis based on Monte Carlo simulations (Lee et.al. 2013), to generate series of samples for each uncertainty factor within a certain range and run the simulations for both January (typical heating dominated month) and July (typical cooling dominated month) and get hourly energy consumptions of the building. The distribution uncertainty for water and air are simulated together since they take places in the same modules. All uncertainties are given the range of $0.8-1.2( \pm 20 \%)$ and run with 50 samples for both January and July except for Water and Air Distribution, which has 33 groups of samples for January and 30 groups of samples for July. The energy consumption differences between the baseline buildings and the test building with each uncertainty are calculated to evaluate the impact of each uncertainty. The results of minimum, maximum and average energy impact of each uncertainty are listed in the tables below. Positive numbers means that the uncertainties bring increases to the energy consumption and negative numbers stand for energy consumption reductions. 
Table 1: Impact of each uncertainty in January.

\begin{tabular}{|c|c|c|c|c|c|}
\hline Uncertainty & $\begin{array}{c}\text { Hourly } \\
\text { Min } \\
(\%)\end{array}$ & $\begin{array}{c}\text { Hourly } \\
\text { Max } \\
(\%)\end{array}$ & $\begin{array}{c}\text { Daily } \\
\text { Min } \\
(\%)\end{array}$ & $\begin{array}{c}\text { Daily } \\
\text { Max } \\
(\%)\end{array}$ & $\begin{array}{c}\text { Monthly } \\
\text { Average } \\
(\%)\end{array}$ \\
\hline $\begin{array}{c}\text { OA } \\
\text { Controller }\end{array}$ & 19.440 & 43.94 & 4.39 & 13.02 & 17.21 \\
\hline Coil UA & -0.002 & 0.002 & - & 0.001 & $<0.001$ \\
\hline $\begin{array}{c}\text { Temperature } \\
\text { Sensor }\end{array}$ & \multicolumn{5}{|c|}{$<0.001$} \\
\hline $\begin{array}{c}\text { Water and } \\
\text { Air } \\
\text { distribution }\end{array}$ & -47.68 & 106.30 & 33.06 & 80.82 & 10.44 \\
\hline
\end{tabular}

Table 2: Impact of each uncertainty in July.

\begin{tabular}{|c|c|c|c|c|c|}
\hline Uncertainty & $\begin{array}{c}\text { Hourly } \\
\text { Min } \\
(\%)\end{array}$ & $\begin{array}{c}\text { Hourly } \\
\text { Max } \\
(\%)\end{array}$ & $\begin{array}{c}\text { Daily } \\
\text { Min } \\
(\%)\end{array}$ & $\begin{array}{c}\text { Daily } \\
\text { Max } \\
(\%)\end{array}$ & $\begin{array}{c}\text { Monthly } \\
\text { Average } \\
(\%)\end{array}$ \\
\hline $\begin{array}{c}\text { OA } \\
\text { Controller }\end{array}$ & -2.96 & 3.5 & -1.29 & 1.44 & 0.55 \\
\hline Coil UA & \multicolumn{5}{|c|}{$<0.001$} \\
\hline $\begin{array}{c}\text { Temperature } \\
\text { Sensor }\end{array}$ & \multicolumn{5}{|c|}{$<0.001$} \\
\hline $\begin{array}{c}\text { Water and } \\
\text { Air } \\
\text { distribution }\end{array}$ & -12.18 & 42.18 & -5.84 & 22.37 & 12.02 \\
\hline
\end{tabular}

Since all the uncertainties are simulated under the same range, it is safe to say that the impacts on energy consumption by uncertainty in heating coil UA and temperature sensors are very small. Deviation introduced by uncertainty in OA controller and in water and air distributions can be significantly higher.

Besides showing the overall impact on energy consumption, the embedded uncertainty modules allow us to check the impact patterns on an hourly base.

Figure 4 below shows the hourly energy consumption distribution for all 50 samples (left y-axis) and the distribution of average hourly consumption difference (right y-axis) for the simulation of test building with
System UQ: OA Controller in January. Figure shows a regularly repeated pattern of energy consumption difference during certain period of time. To look at the values in a more explicit way, 4 groups of values of hourly energy consumption difference for 4 typical days in January are shown as below in color scaled cells, in which red represents top $10 \%$ highest value, blue represents $10 \%$ lowest value, and yellow represents 0 .

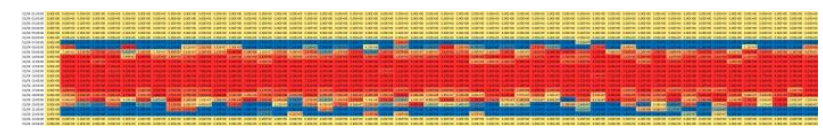

Figure 5: Hourly Energy Consumption Difference for January $4^{\text {th }}$.

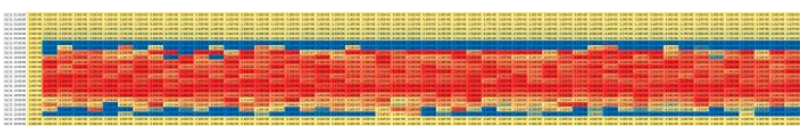

Figure 6: Hourly Energy Consumption Difference for January $11^{\text {th }}$.

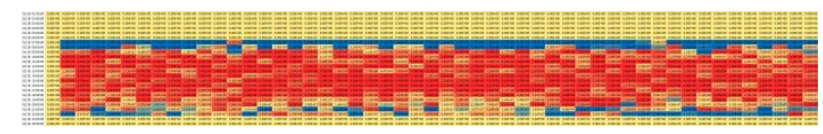

Figure 7: Hourly Energy Consumption Difference for January $18^{\text {th }}$.

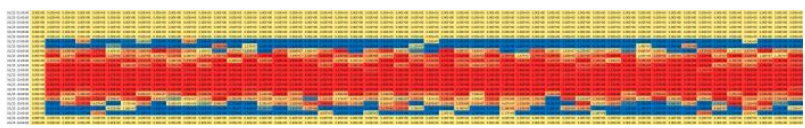

Figure 8: Hourly Energy Consumption Difference for January $25^{\text {th }}$.

Figure 5 to 8 shows that the test building has higher energy consumption then the baseline from 12:00:00 to 18:00:00 during the days, and has lower energy consumption during mornings and nights. This pattern is reasonable since Atlanta has relative warm winter days, and the outdoor air temperature in the afternoon may meet the requirements and trigger the economizer to bring in more outdoor air, which gives OA controller

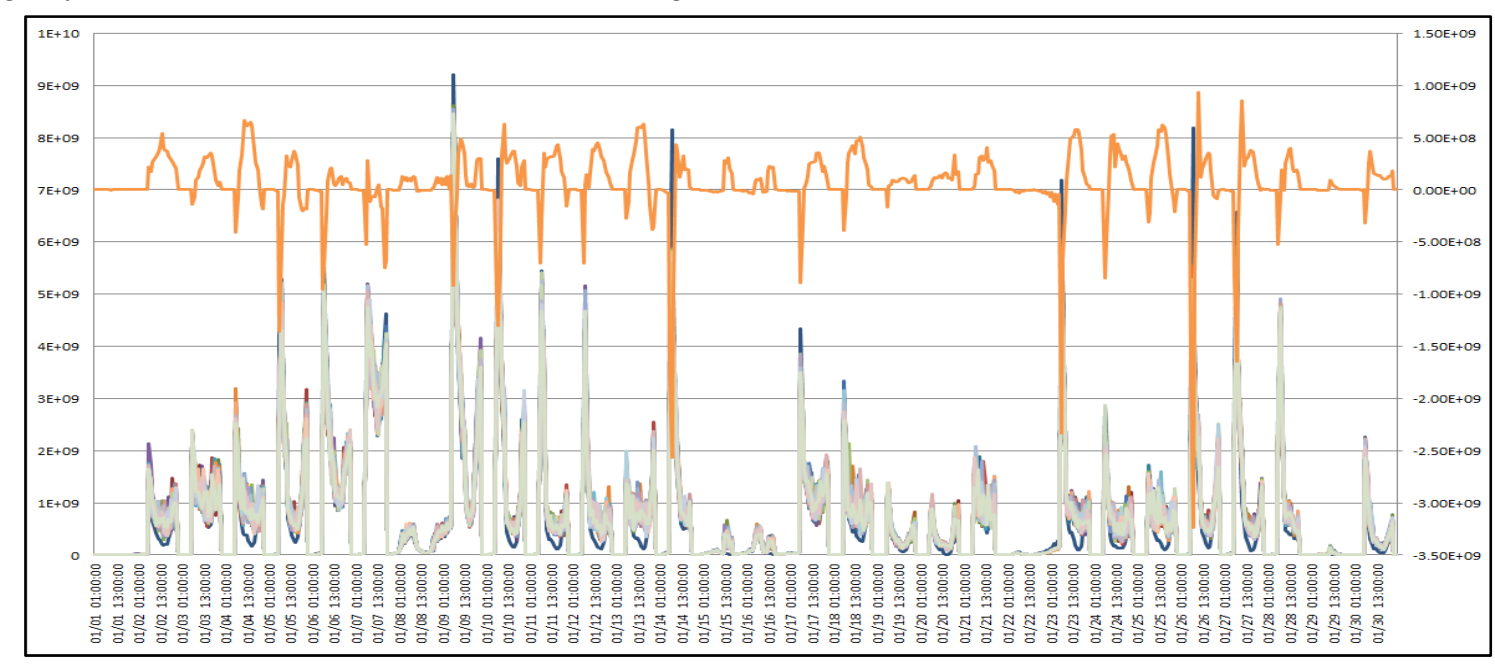

Figure 4: Hourly Energy Consumption and Hourly Average Energy Consumption Difference Distributions for UQ: OA Controller, January. 
more space to affect the energy consumption.

\section{Conclusion and future work}

This study investigates the features of 5 HVAC operation uncertainties detected in the case building, generates methods that can capture the impacts of those uncertainties, upgrades EnergyPlus by updating its source code, eventually testing the impacts of the 5 uncertainties using GURA-W by UA based on sampling over a hypothetical uncertainty range which is taken as $\pm 20 \%$, using the extended version of EnergyPlus with upgraded AHU modules. The study presents a feasible way to embed the uncertainty calculation into an existing simulation tool (EnergyPlus) which is easier and more practical to use for actual building design and analysis. In addition to the practicability, compared to applying a general assumption on uncertainties over the total model result, this modified tool can better assist design teams in decision makings by allowing projects to simulate the energy impact of each uncertainty factor individually. For example, if the impact is considered small enough to be ignored, the related equipment could be selected with lower precision.

The next step of this work is to apply those system operation uncertainties to real simulations. But some issues deserve special attention:

\section{Limitations}

For the heating coil UA factor, our treatment has only shown how to add UA factor uncertainty to heating coils and not cooling coils. As cooling coil play an important role in cooling mode, it is necessary to also investigate how EnergyPlus models cooling coil and add UA uncertainty to it.

For temperature sensor uncertainty, it would be better to relocate the sensor uncertainty to zone temperature sensor, as we addressed in the last chapter.

For water distribution, as it was introduced in the last chapter, our upgraded EnergyPlus model cannot model the unbalancing of AHU coils and VAV reheat coils together (as addressed at the end of the last chapter). To connect those two types of coils together, one needs to loop over Manage HVAC twice and make sure nothing else will be affected or find another way to capture the uncertainty.

We only considered 5 uncertainties, while there still a lot of additional operation uncertainties exist in the system, for instance, the uncertainty of supply air leakage, and uncertainties caused by boiler and chiller operation.

\section{Applicability to other buildings}

Since the uncertainties are caused by HVAC operation, which are specific to the HVAC system, the specifics of the building will not have a significant influence factor on the operation uncertainty. Thus, once the uncertainties have been well captured, we can use them in the simulation of any building with the same HVAC system type.

Future work: Quantification of the uncertainty ranges in uncertainty analysis of building designs

As the uncertainties are mainly affected by HVAC system type, we can collect data from a set of monitored buildings with the same HVAC system type and calibrate the uncertainty ranges over the set of buildings. This requires selection of suitable buildings with appropriate metered data.

The ultimate objective of the work will be to do a full uncertainty analysis of building energy models and determine through sensitivity analysis what the role is of the system parameters on the resulting energy performance. This will lead to generic statements about the need to consider system uncertainty as part of a simulation and the need to make system upgrades part of a planned retrofit.

\section{References}

L. Wang, P. Mathew, X. Pang (2012). Uncertainties in energy consumption introduced by building operations and weather for a medium-size office building, Energy and Buildings, 2012.

B. Yan and A., Malkawi (2012). Predicting System Performance with Uncertainty, In Proceedings of the Twelfth International Conference for Enhanced Building Operations (ICEBO) Held in Manchester, the United Kingdom, 23-26, October 2012. Manchester, the United Kingdom.

M. Basarkar, X. Pang, L. Wang, P. Haves, T. Hong (2011). Modeling and Simulation of HVAC Faults in EnergyPlus, In Proceeding of Building Simulation 2011: 12th Conference of International Building Performance Simulation Assocication, Sydney, 14-16 November.

EnergyPlus (2011). Guide for Module Developers, Orlando: Ernest Orlando Lawrence Berkeley National Laboratory.

EnergyPlus (2011). EnergyPlus Engineering Reference, Orlando: Ernest Orlando Lawrence Berkeley National Laboratory.

X. Zhang (2001). Heat Transfer (4th Edition). Beijing: China Architechture and Building Press, 2001. Print.

B.D. Lee, Y. Sun, G. Augenbroe, C.J.J. Paredis (2013). Toward Better Prediction of Building Performance: A Workbench to Analyze Uncertainty in Building Simulation, in, 13th International Building Performance Simulation Association Conference(To be Published), 2013. 